\title{
Immunological Factors in Pompe Disease Management: Clinical Experience and Implications for Newborn Screening
}

\author{
Priya S. Kishnani ${ }^{1, *}$, Zoheb B. Kazi ${ }^{1}$, Kathryn L. Berrier ${ }^{1}$, Stephanie M. Dearmey ${ }^{1}$, Deeksha S. Bali ${ }^{1}$, \\ Amy S. Rosenberg ${ }^{2}$ \\ ${ }^{1}$ Division of Medical Genetics, Department of Pediatrics, Duke University Health System, Durham, NC \\ ${ }^{2}$ Division of Therapeutic Proteins, Office of Biotechnology Products, Center for Drug Evaluation and Research, \\ United States Food and Drug Administration
}

\section{BACKGROUND}

Enzyme replacement therapy (ERT) with alglucosidase alfa has improved clinical outcomes and prolonged survival for patients with infantile Pompe disease (IPD). However, patients characterised as Cross-Reactive Immunological Material (CRIM)negative $(\mathrm{CN})$ mount an immune response against ERT resulting in clinical decline and, ultimately, death. A prophylactic immune tolerance induction (ITI) protocol has been successful in preventing the development of antibody titres for these patients. A subset of (CP) patients also mount an immune response with poor clinical outcomes, these at-risk patients are difficult to identify. With the advent of newborn screening for Pompe disease, preventative treatment to circumvent an immune response is warranted; therefore, our study aimed to assess the safety and efficacy of prophylactic ITI in the naïve setting for both CP and CN IPD patients.

\section{MATERIALS AND METHODS}

This was a retrospective chart review of a large cohort of IPD patients enrolled in Institutional Review Board (IRB)-approved protocol 00001562 [LDN6709 Site 206; Clinicaltrials.gov Identifier: NCT01665326] for CRIM determination and longitudinal follow-up. Baseline and follow-up data were obtained to assess treatment outcomes including age at diagnosis and initiation of ERT, ventilator-free survival and cardiac function.

\footnotetext{
*Correspondence to: Priya S. Kishnani, MD, Duke University Medical Center, GSRB1 Box 103856, 4th floor, 905 S. LaSalle Street, Durham, North Carolina 27710 USA, Tel.: +1 919684 2036; Fax: +1 919 668-8944. E-mail: priya.kishnani@duke.edu.
}

\section{RESULTS}

Prophylactic ITI treatment was administered in the naïve setting for $26 \mathrm{CN}$ patients-involving rituximab, methotrexate and IV IG, 6 patients with prior exposure to ERT and development of high sustained antibody titres and 4 high-risk CP patients. While most patients needed only one round of prophylactic ITI, 3 required a second round due to the presence of anti-rhGAA antibody titres. Overall, patients that have completed the ITI regimen are currently immune tolerant. The oldest CRIM-negative survivor is 9 years old. Aside from one instance of transient low neutrophil count, there were no serious adverse events related to ITI medications.

\section{CONCLUSIONS}

Currently, ITI addresses immune issues for only $30 \%$ of the IPD population- $\mathrm{CN}$ patients; yet, a significant proportion of $\mathrm{CP}$ patients are also at risk for an immune response. With the advent of newborn screening for Pompe disease, early identification and pre-emptive treatment is essential. Our results represent the largest cohort of IPD cases treated with prophylactic ITI with promising results, which emphasise the need to seriously consider ITI as standard of care in the management of all IPD patients. Given the overall safety of ITI, universal implementation of ITI for both CP and CN IPD patients is warranted. Because the majority of IPD patients develop antibodies within the first month of ERT, a mechanism to identify at-risk patients would help to effectively guide prophylactic ITI in the naïve setting. 